\title{
Magnetic Particle Imaging for Magnetic Hyperthermia Treatment: Visualization and Quantification of the Intratumoral Distribution and Temporal Change of Magnetic Nanoparticles in Vivo
}

\author{
Tomomi Kuboyabu, Isamu Yabata, Marina Aoki, Natsuo Banura, Kohei Nishimoto, \\ Atsushi Mimura, Kenya Murase* \\ Department of Medical Physics and Engineering, Division of Medical Technology and Science, Faculty of Health \\ Science, Graduate School of Medicine, Osaka University, Osaka, Japan \\ Email: "murase@sahs.med.osaka-u.ac.jp
}

Received 20 January 2016; accepted 14 March 2016; published 17 March 2016

Copyright (C) 2016 by authors and Scientific Research Publishing Inc.

This work is licensed under the Creative Commons Attribution International License (CC BY).

http://creativecommons.org/licenses/by/4.0/

c) (i) Open Access

\begin{abstract}
Purpose: Magnetic hyperthermia treatment (MHT) is a strategy for cancer therapy using the temperature rise of magnetic nanoparticles (MNPs) under an alternating magnetic field (AMF). Recently, a new imaging method called magnetic particle imaging (MPI) has been introduced. MPI allows imaging of the spatial distribution of MNPs. The purpose of this study was to investigate the feasibility of visualizing and quantifying the intratumoral distribution and temporal change of MNPs and predicting the therapeutic effect of MHT using MPI. Materials and Methods: Colon-26 cells $\left(1 \times 10^{6}\right.$ cells $)$ were implanted into the backs of eight-week-old male BALB/c mice. When the tumor volume reached approximately $100 \mathrm{~mm}^{3}$, mice were divided into untreated (n = 10) and treated groups $(n=27)$. The tumors in the treated group were directly injected with MNPs (Resovist $\left.{ }^{\circledR}\right)$ with iron concentrations of $500 \mathrm{mM}(A, n=9), 400 \mathrm{mM}(B, n=8)$, and $250 \mathrm{mM}(C, n=10)$, respectively, and MHT was performed using an AMF with a frequency of $600 \mathrm{kHz}$ and a peak amplitude of $3.5 \mathrm{kA} / \mathrm{m}$. The mice in the treated group were scanned using our MPI scanner immediately before, immediately after, 7 days, and 14 days after MHT. We drew a region of interest (ROI) on the tumor in the MPI image and calculated the average, maximum, and total MPI values and the number of pixels by taking the threshold value for extracting the contour as $40 \%$ of the maximum MPI value (pixel value) within the ROI. These parameters in the untreated group were taken as zero. We also measured the relative tumor volume growth (RTVG) defined by $\left(V-V_{0}\right) / V_{0}$, where $V_{0}$ and $V$ are the tumor volumes immediately before and after MHT, respectively. Results:
\end{abstract}

\footnotetext{
${ }^{*}$ Corresponding author.

How to cite this paper: Kuboyabu, T., Yabata, I., Aoki, M., Banura, N., Nishimoto, K., Mimura, A. and Murase, K. (2016) Magnetic Particle Imaging for Magnetic Hyperthermia Treatment: Visualization and Quantification of the Intratumoral Distribution and Temporal Change of Magnetic Nanoparticles in Vivo. Open Journal of Medical Imaging, 6, 1-15.

http://dx.doi.org/10.4236/ojmi.2016.61001
} 
The average, maximum, and total MPI values decreased up to 7 days after MHT and remained almost constant thereafter in all groups, whereas the number of pixels tended to increase with time. The RTVG values in Groups A and B were significantly lower than those in the control group 3 days or more and 5 days or more after MHT, respectively. The above four parameters were significantly inversely correlated with the RTVG values 5, 7, and 14 days after MHT. Conclusion: MPI can visualize and quantify the intratumoral distribution and temporal change of MNPs before and after MHT. Our results suggest that MPI will be useful for predicting the therapeutic effect of MHT and for the treatment planning of MHT.

\section{Keywords} Magnetic Particle Imaging, Magnetic Hyperthermia Treatment, Magnetic Nanoparticles,
Intratumoral Distribution, Temporal Change

\section{Introduction}

Hyperthermia is one approach to cancer therapy, and is based on the fact that cancer cells are more sensitive to heat than normal tissues. The most commonly used heating method in the clinical setting is capacitive heating that uses a radiofrequency (RF) electric field [1]. The therapeutic effect of hyperthermia depends on the temperature of the targeted region and the heating duration [2]. The cell-killing mechanism of hyperthermia is related to the activation of immune system, and its efficacy increases dramatically at temperatures above $42^{\circ} \mathrm{C}$ to $43^{\circ} \mathrm{C}$ [3]. The therapeutic effect of hyperthermia can also be enhanced by combining it with radiotherapy, chemotherapy, and immunotherapy [4]-[6]. Conventional hyperthermia treatments, however, cause damage to not only cancer cells but also normal tissues. Therefore, it is important to heat the targeted region selectively for safe treatment.

Magnetic hyperthermia treatment (MHT) is one of the methods for hyperthermia treatment and employs the temperature rise of magnetic nanoparticles (MNPs) under an alternating magnetic field (AMF). MNPs generate heat through hysteresis loss and/or relaxational loss when exposed to AMF [7]. MHT can selectively heat tumor cells without damaging normal tissues [8]. In order to enhance the therapeutic effect of MHT, it is necessary to deliver and accumulate as many MNPs as possible into the tumor tissues [9]. Therefore, the development of functionalized MNPs to improve their delivery, accumulation, and anti-tumor effect has attracted recent attention. In addition, various nanocarriers such as magnetic liposomes loading anti-tumor agents, magnetic cationic liposomes, and antibody-conjugated magnetic liposomes have been developed for more effective cancer treatment in animal models [10]-[12].

Magnetic particle imaging (MPI) is an imaging method that has been introduced recently [13]. MPI uses a nonlinear response of MNPs to an external oscillating magnetic field and is capable of imaging the spatial distribution of MNPs such as superparamagnetic iron oxide (SPIO) with high sensitivity and high spatial resolution [13].

The purpose of this study was to investigate the feasibility of visualizing and quantifying the intratumoral distribution and temporal change of MNPs using MPI and to evaluate the usefulness of MPI for predicting the therapeutic effect of MHT.

\section{Materials and Methods}

\subsection{System for Magnetic Particle Imaging}

The details of our MPI system are described in our previous papers [14]-[19]. In brief, a drive magnetic field was generated using an excitation coil (solenoid coil $100 \mathrm{~mm}$ in length, $80 \mathrm{~mm}$ in inner diameter, and $110 \mathrm{~mm}$ in outer diameter). AC power was supplied to the excitation coil by a programmable power supply (EC1000S, NF CO., Yokohama, Japan), and was controlled using a sinusoidal wave generated by a digital function generator (DF1906, NF Co., Yokohama, Japan). The frequency and peak-to-peak strength of the drive magnetic field were taken as $400 \mathrm{~Hz}$ and $20 \mathrm{mT}$, respectively. The signal generated by MNPs was received by a gradiometer 
coil (50 mm in length, $35 \mathrm{~mm}$ in inner diameter, and $40 \mathrm{~mm}$ in outer diameter), and the third-harmonic signal was extracted using a preamplifier (T-AMP03HC, Turtle Industry Co., Ibaragi, Japan) and a lock-in amplifier (LI5640, NF Co., Yokohama, Japan). The output of the lock-in amplifier was converted to digital data by a personal computer connected to a multifunction data acquisition device with a universal serial bus port (USB-6212, National Instruments Co., TX, USA). The sampling time was taken as $10 \mathrm{msec}$. When measuring signals using the gradiometer coil, a sample was placed $12.5 \mathrm{~mm}$ (i.e., one quarter of the coil length) from the center of the gradiometer coil and the coil, including the sample, was moved such that the center of the sample coincided with the position of a field-free line. The selection magnetic field was generated by two opposing neodymium magnets (Neomax Engineering Co., Gunma, Japan). The field-free line can be generated at the center of the two neodymium magnets.

To acquire projection data for image reconstruction, a sample in the receiving coil was automatically rotated around the z-axis over $180^{\circ}$ in steps of $5^{\circ}$ and translated in the $\mathrm{x}$-direction from $-16 \mathrm{~mm}$ to $16 \mathrm{~mm}$ in steps of 1 mm, using an XYZ-axes rotary stage (HPS80-50X-M5, Sigma Koki Co., Tokyo, Japan) controlled by LabVIEW (National Instruments Co., TX, USA). Data acquisition took about 12 min. Each projection data set was then transformed into 64 bins by linear interpolation. Both the inhomogeneous sensitivity of the receiving coil and feed through interference were corrected using the method described in [16]. Transverse images were reconstructed from the projection data using the maximum likelihood-expectation maximization (ML-EM) algorithm over 15 iterations, in which the initial concentration of MNPs was assumed to be uniform [14] [17].

\subsection{System for Magnetic Hyperthermia Treatment}

The details of our apparatus for MHT are described in our previous papers [7] [15]. In brief, the coil for generating the AMF consists of 19-turned loops (6.5 $\mathrm{cm}$ in diameter and $10 \mathrm{~cm}$ in length) of copper pipe (5 mm in diameter) cooled by water to ensure constant temperature and impedance. The coil was connected to a highfrequency power supply (T162-5723BHE, Thamway Co., Ltd., Shizuoka, Japan) and a manual-matching unit (T020-5723AHE, Thamway Co., Ltd., Shizuoka, Japan). This system can induce an AMF with a maximum peak amplitude of $3.7 \mathrm{kA} / \mathrm{m}$ at an output power of $500 \mathrm{~W}$. The peak amplitude of the AMF generated in the coil can be controlled by changing the output of the power supply.

\subsection{Tumor-Bearing Mice and Magnetic Nanoparticles}

Colon-26 cells (a mouse cell line derived from rectal cancer) (RIKEN BioResource Center, Ibaraki, Japan) were cultured in RPMI-1640 medium (Mediatech Inc., VA, USA) supplemented with 10\% fetal calf serum (Biowest, Nuaillé, France) and 1\% penicillin-streptomycin (Nacalai Tesque Inc., Kyoto, Japan). All cultures were incubated in a humidified atmosphere containing $5 \% \mathrm{CO}_{2}$ at $37^{\circ} \mathrm{C}$. The cells were trypsinized with $0.25 \%$ trypsin in ethylenediaminetetraacetic acid (EDTA) (Nacalai Tesque Inc., Kyoto, Japan) and resuspended in phosphate-buffered saline (PBS) at $1 \times 10^{6}$ cells/100 $\mu \mathrm{L}$. The cells $\left(1 \times 10^{6}\right.$ cells $)$ were implanted into the backs of eight-week-old male BALB/c mice (Charles Liver Laboratories Japan, Inc., Yokohama, Japan) on the same day and under the same conditions. During the implantation, the mice were anesthetized by intraperitoneal administration of pentobarbital sodium (Somnopentyl, Kyoritsu Seiyaku Co., Tokyo, Japan) (10-fold dilution, 0.012 $\mathrm{mL} / \mathrm{g}$ body weight).

In this study, Resovist ${ }^{\circledR}$ (FUJIFILM RI Pharma Co., Ltd., Tokyo, Japan) was used as the source of MNPs. Resovist $^{\circledR}$ is an organ-specific contrast agent for magnetic resonance imaging, used especially for the detection and characterization of small focal liver lesions [7] [15]. It consists of MNPs (maghemite, $\gamma-\mathrm{Fe}_{2} \mathrm{O}_{3}$ ) coated with carboxydextran.

\subsection{Study Protocol}

When the tumor volume had grown to approximately $100 \mathrm{~mm}^{3}$, mice were divided into a control group $(\mathrm{n}=10)$ and three treatment groups (A, B, and C). The mice in the control group were not treated with MHT. The tumors in the mice in Groups A $(n=9), B(n=8)$, and C $(n=10)$ were directly injected with Resovist ${ }^{\circledR}(0.2 \mathrm{~mL}$ of stock solution or stock solution diluted in PBS) with iron concentrations of $500 \mathrm{mM}, 400 \mathrm{mM}$, and $250 \mathrm{mM}$, respectively, under anesthesia. After injection of Resovist ${ }^{\circledR}$, each mouse was placed in a plastic holder for undergoing MPI and MHT. 
Figure 1 illustrates the protocol for data acquisition. As illustrated in Figure 1, MHT was started 20 min after the injection of Resovist ${ }^{\circledR}$. MHT was performed by applying an AMF at a frequency of $600 \mathrm{kHz}$ and a peak amplitude of $3.5 \mathrm{kA} / \mathrm{m}$ [16] for $20 \mathrm{~min}$. During MHT, the temperatures of the tumor and rectum were recorded using two fluorescence-type optical fiber thermometers (AMOTH FL-2000, Anritsu Meter Co., Tokyo, Japan) and two optical fiber temperature probes. One probe was placed in the tumor and the other probe was inserted 1 $\mathrm{cm}$ inside the rectum. Both temperatures were recorded every second until the end of MHT.

The MPI studies were performed four times for each mouse; immediately before MHT (2 min after the injection of Resovist ${ }^{\circledR}$ ), immediately after MHT (42 min after the injection of Resovist ${ }^{\circledR}$ ) and 7 days and 14 days after MHT (Figure 1). After the MPI studies, X-ray CT images were obtained using a 4-row multi-slice CT scanner (Asteion, Toshiba Medical Systems Co., Tochigi, Japan) with a tube voltage of $120 \mathrm{kV}$, a tube current of 210 $\mathrm{mA}$, and a slice thickness of $0.5 \mathrm{~mm}$. The MPI image was co-registered to the X-ray CT image using the method described in [15]. It should be noted that the X-ray CT image after the second MPI study was substituted by that obtained after the first MPI study.

All animal experiments described above were approved by the animal ethics committee at Osaka University School of Medicine.

\subsection{Data and Statistical Analyses}

The dimensions of the tumor were measured with a caliper every day and the tumor volume ( $V$ ) was calculated from $V=(\pi / 6) \times L_{x} \times L_{y} \times L_{z}$, where $L_{x}, L_{y}$, and $L_{z}$ represent the vertical diameter, horizontal diameter, and height in mm, respectively. The relative tumor volume growth (RTVG) was also calculated from $\left(V-V_{0}\right) / V_{0}$, where $V_{0}$ represents the tumor volume immediately before MHT. In this study, the RTVG value was used as an indicator of the therapeutic effect of MHT.

We drew a region of interest (ROI) on the tumor in the MPI image and calculated the average, maximum, and total MPI values by taking the threshold value for extracting the contour of the tumor as $40 \%$ of the maximum MPI value within the ROI. In this study, the MPI value was defined as the pixel value of the transverse MPI image reconstructed from the third-harmonic signals. We also calculated the number of pixels within the ROI. It should be noted that the total MPI value is equal to the product of the average MPI value and the number of pixels. The above parameters in the control group were taken as zero.

The temperature, RTVG, average MPI value, maximum MPI value, total MPI value, and the number of pixels were expressed as the mean \pm standard error (SE). Differences in these parameters among groups were analyzed by one-way analysis of variance (ANOVA). Statistical significance was determined by Tukey’s multiple comparison test. A $P$ value less than 0.05 was considered statistically significant.

\section{Results}

Figure 2 shows the time courses of the temperature in the tumor and rectum during MHT in Groups A (red circles), B (blue circles), and $\mathrm{C}$ (green circles). The temperature in the rectum was almost constant at approximately $35^{\circ} \mathrm{C}$ five minutes or more after the start of MHT. The temperature in the tumor before MHT was $31^{\circ} \mathrm{C}$ to $34^{\circ} \mathrm{C}$. The temperature in the tumor increased after the start of MHT and plateaued at approximately $44^{\circ} \mathrm{C}, 42^{\circ} \mathrm{C}$, and $41^{\circ} \mathrm{C}$ in Groups A, B, and C, respectively. The maximum temperature in the tumor became higher with increasing iron concentration.

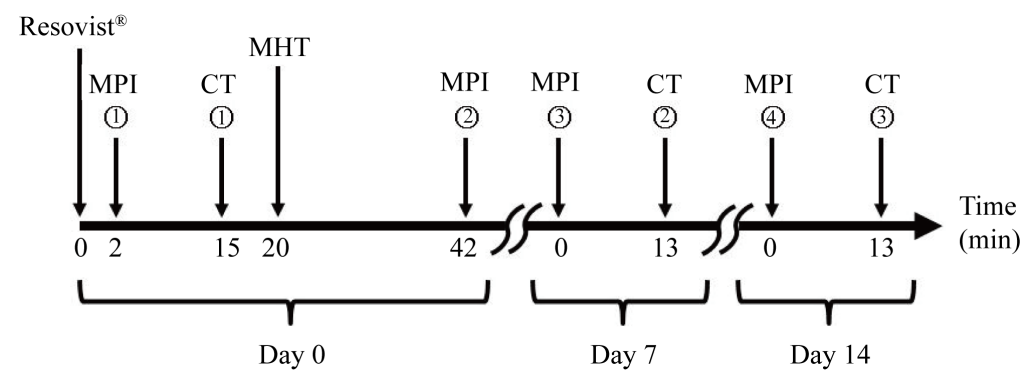

Figure 1. Protocol for data acquisition. MPI: magnetic particle imaging, CT: X-ray computed tomography, and MHT: magnetic hyperthermia treatment. 


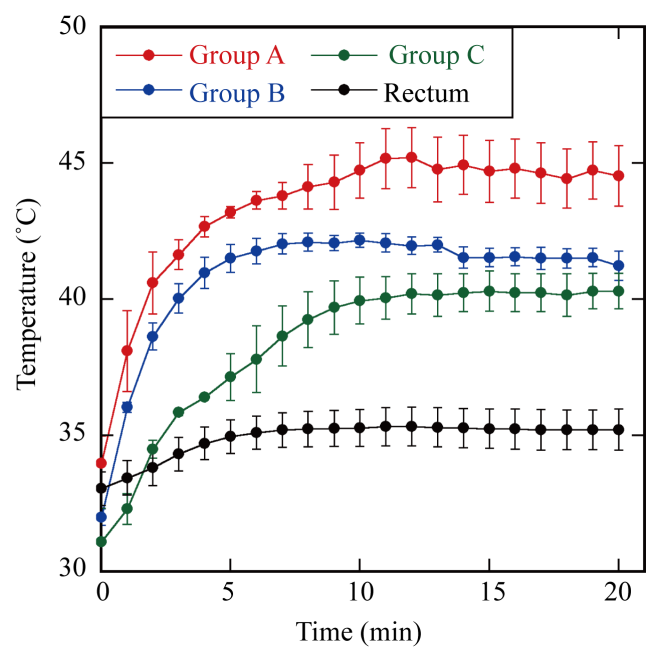

Figure 2. Time courses of the temperature in the tumor and rectum of the mice in Groups A (red circles), B (blue circles), and $\mathrm{C}$ (green circles) during MHT for $20 \mathrm{~min}$. The tumors in Groups A, B, and C were directly injected with Resovist ${ }^{\circledR}$ with iron concentrations of $500 \mathrm{mM}, 400 \mathrm{mM}$, and $250 \mathrm{mM}$, respectively. Data are represented by mean \pm standard error (SE).

Figure 3 shows the RTVG values as a function of days after MHT in Groups A (red circles), B (blue circles), and C (green circles), and the control group (black circles). The RTVG value decreased with increasing iron concentration. The RTVG value in Group A was significantly lower than that in the control group 3 days or more after MHT. The RTVG value in Group B was significantly lower than that in the control group 5 days or more after MHT. The RTVG value in Group C was significantly lower than that in the control group 5 days after MHT.

Figure 4 shows the MPI images in Groups A, B, and C immediately before (upper row), immediately after (second row), 7 days after (third row), and 14 days after MHT (bottom row). Note that the MPI images were superimposed on the X-ray CT images. As shown in Figure 4, the MPI value decreased and the spatial distribution of MNPs changed with time.

Figure 5(a) shows the average MPI values immediately before (red bar), immediately after (blue bar), 7 days after (green bar), and 14 days after MHT (black bar) in Groups A, B, and C. Figures 5(b)-(d) show the cases for maximum MPI value, total MPI value, and number of pixels, respectively. The asterisks in the graphs indicate statistical significance $(P<0.05)$. The average, maximum, and total MPI values decreased greatly between immediately before and immediately after MHT and between immediately after and 7 days after MHT and remained almost constant thereafter in all groups (Figures 5(a)-(c)). In contrast, the number of pixels tended to increase with time in Groups A and C (Figure 5(d)). Although the same tendency was also observed in Group B, there were no combinations with significant difference due to large scattering of the data.

Figure 6 shows the correlations between the average MPI value immediately before MHT and the RTVG value (left column) and between the average MPI value immediately after MHT and the RTVG value (right column). The upper, middle, and lower rows show cases when the RTVG values at 5, 7, and 14 days after MHT were used, respectively. The correlation coefficients between the average MPI value immediately before MHT and the RTVG value were $-0.696,-0.666$, and -0.642 at 5,7 , and 14 days after MHT, respectively, whereas those between the average MPI value immediately after MHT and the RTVG value were $-0.658,-0.667$, and -0.650 at 5 , 7, and 14 days after MHT, respectively.

Figure 7 shows the correlations between the maximum MPI value immediately before MHT and the RTVG value (left column) and between the maximum MPI value immediately after MHT and the RTVG value (right column). As in Figure 6, the upper, middle, and lower rows show the cases when the RTVG values at 5, 7, and 14 days after MHT were used, respectively. The maximum MPI value immediately before MHT was significantly inversely correlated with the RTVG value $(r=-0.656,-0.630$, and -0.623 at 5,7 , and 14 days after MHT, respectively). The maximum MPI value immediately after MHT was also significantly inversely correlated with the RTVG value ( $r=-0.662,-0.671$, and -0.651 at 5 , 7 , and 14 days after MHT, respectively). 


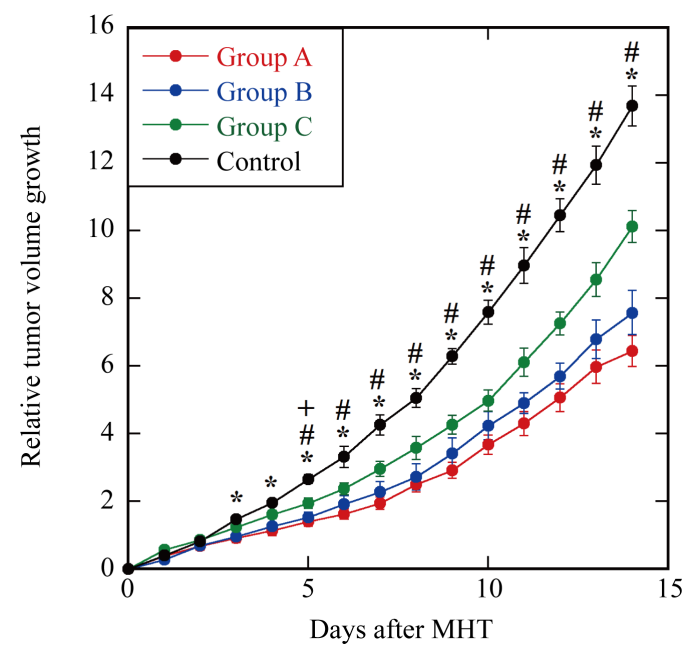

Figure 3. Relative tumor volume growth (RTVG) as a function of days after MHT in Groups A (red circles), B (blue circles), and C (green circles) and the control group (black circles). The RTVG was calculated from $\left(V-V_{0}\right) / V_{0}$, where $V_{0}$ and $V$ represent the tumor volumes immediately before and after MHT, respectively. Note that the mice in the control group were not treated with MHT. Data are represented by mean $\pm \mathrm{SE}$. $* P<0.05$ between Group $\mathrm{A}$ and the control group, $\# P<0.05$ between Group $B$ and the control group, and + $P<0.05$ between Group $\mathrm{C}$ and the control group.

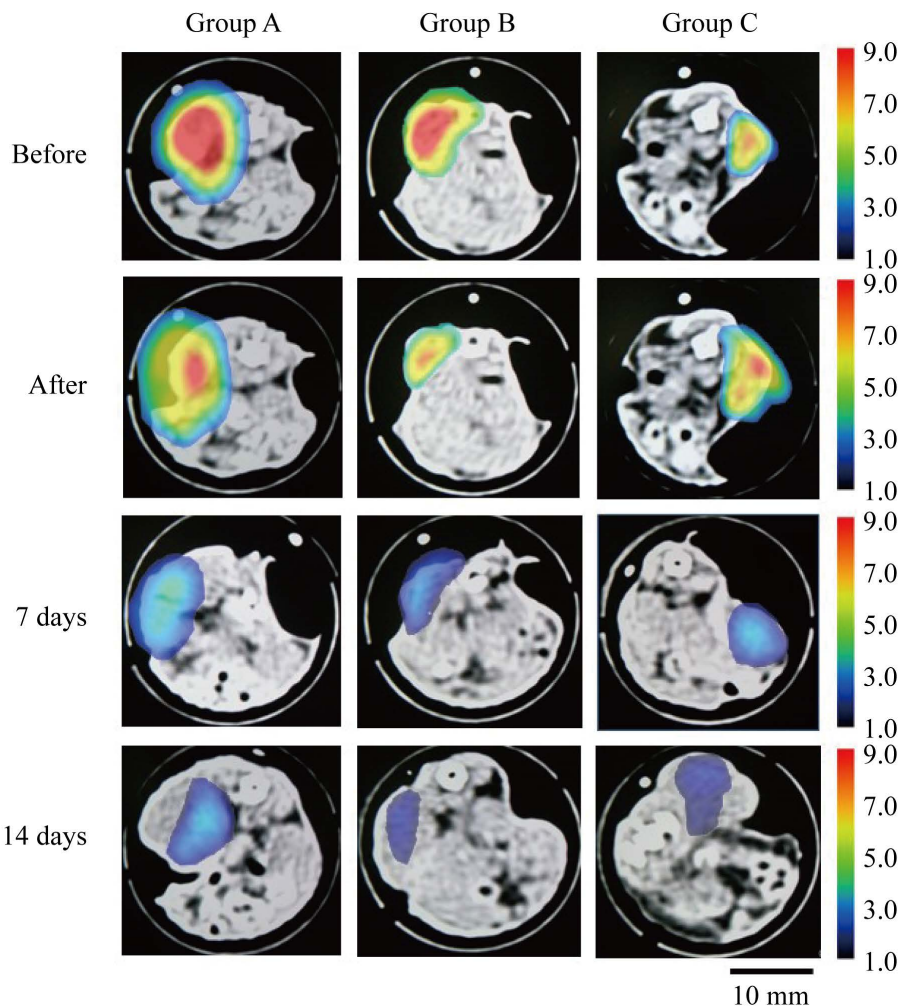

Figure 4. Examples of the MPI images in Groups A, B, and C immediately before (upper row), immediately after (second row), 7 days (third row), and 14 days after MHT (bottom row). Note that the MPI images were superimposed on the X-ray CT images. Scale bar $=10 \mathrm{~mm}$. 


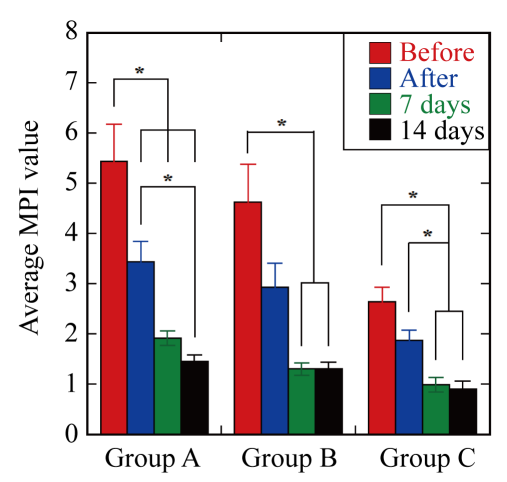

(a)

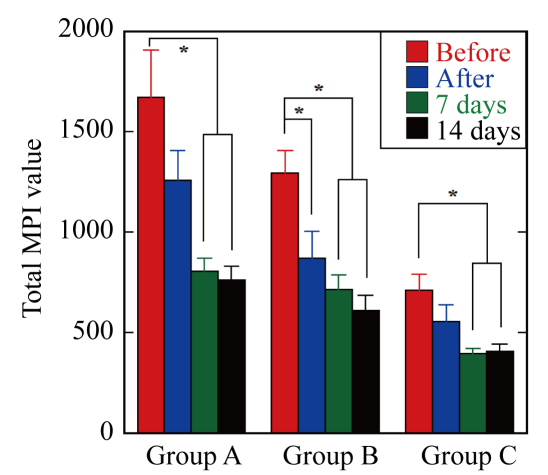

(c)

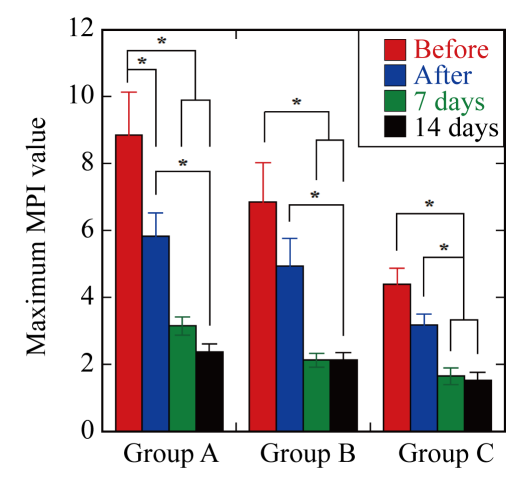

(b)

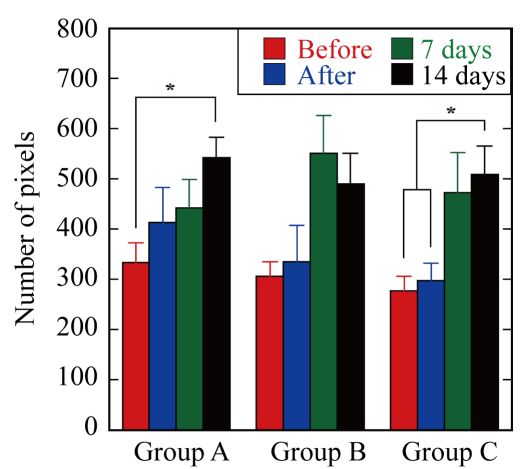

(d)

Figure 5. Average MPI values (a), maximum MPI values (b), total MPI values (c), and the numbers of pixels within the region of interest (ROI) drawn on the tumor in the MPI image (d) immediately before, immediately after, 7 days, and 14 days after MHT in Groups A, B, and C. Bar and error bar represent mean and SE, respectively. $* P<0.05$.

Figure 8 shows cases for the total MPI value. The total MPI value immediately before MHT was significantly inversely correlated with the RTVG value $(r=-0.702,-0.669$, and -0.640 at 5,7 , and 14 days after MHT, respectively). The total MPI value immediately after MHT was also significantly inversely correlated with the RTVG value ( $r=-0.593,-0.608$, and -0.553 at 5,7 , and 14 days after MHT, respectively).

Figure 9 shows cases for the number of pixels. The number of pixels immediately before MHT was significantly inversely correlated with the RTVG value $(r=-0.629,-0.571$, and -0.507 at 5 , 7 , and 14 days after MHT, respectively). The number of pixels immediately after MHT was also significantly inversely correlated with the RTVG value ( $r=-0.581,-0.517$, and -0.392 at 5,7 , and 14 days after MHT, respectively).

\section{Discussion}

We previously demonstrated that the average MPI value has excellent correlations with the iron concentration of MNPs and the temperature rise of Resovist ${ }^{\circledR}$ solution induced by AMF [15]. We also reported the preliminary results of in vivo studies on the application of MPI to MHT [15]. In this study, we evaluated the feasibility of visualizing and quantifying the intratumoral distribution and temporal change of MNPs in vivo using MPI and investigated the usefulness of MPI for predicting the therapeutic effect of MHT.

In this study, we investigated the therapeutic effect of MHT using tumor-bearing mice injected intratumorally with Resovist ${ }^{\circledR}$ with iron concentrations of $500 \mathrm{mM}, 400 \mathrm{mM}$, and $250 \mathrm{mM}$. According to Yonezawa et al. [20], cancer cells undergo apoptosis when exposed to heat shock at $42^{\circ} \mathrm{C}$ to $43^{\circ} \mathrm{C}$, whereas hyperthermia at temperatures above $44^{\circ} \mathrm{C}$ causes necrosis. Mild hyperthermia at approximately $40^{\circ} \mathrm{C}$, however, decreases the induction of cytotoxicity and endoplasmic reticulum (ER) stress and rather protects cells against ER stress-induced 


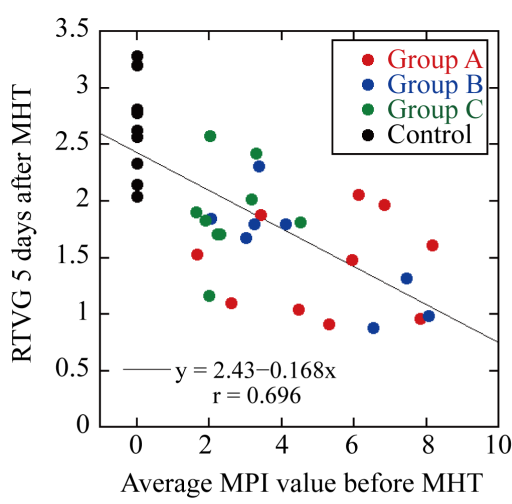

(a)

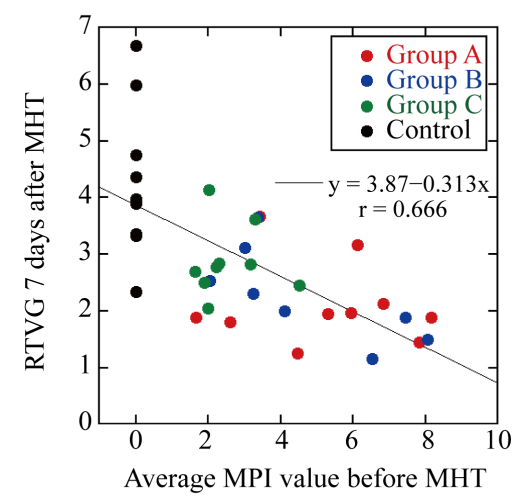

(c)

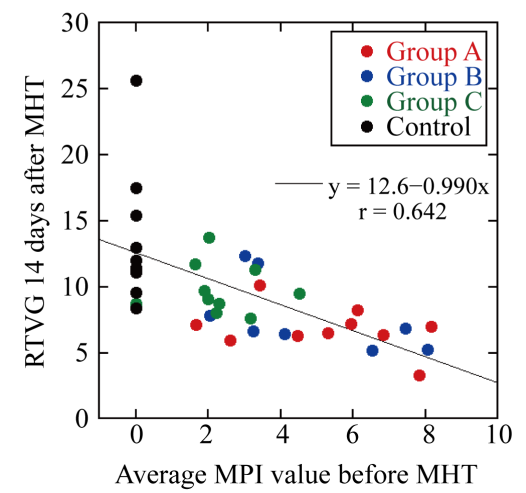

(e)

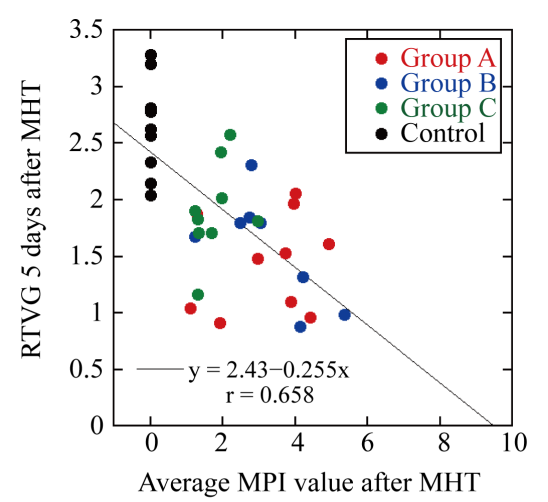

(b)

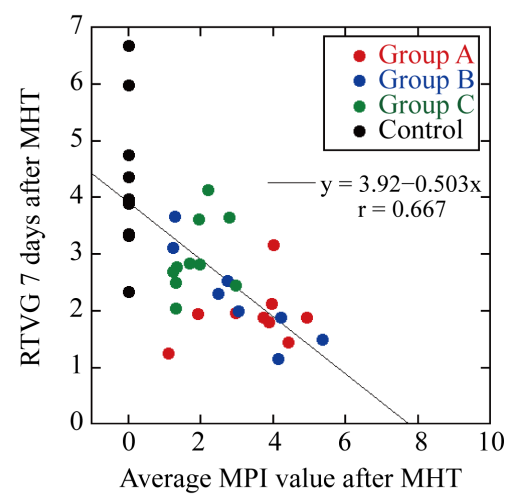

(d)

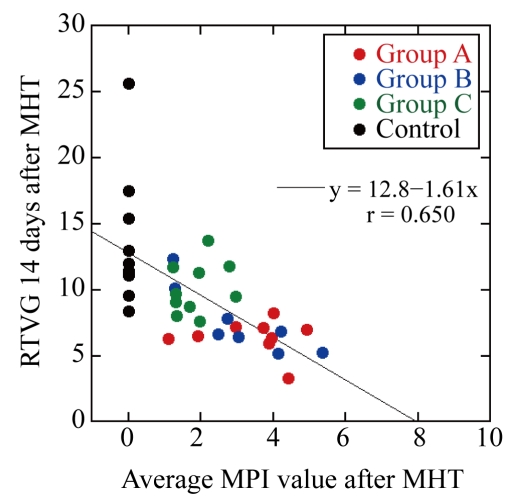

(f)

Figure 6. Correlation between the average MPI and RTVG values. The left and right columns show cases when the average MPI values immediately before and immediately after MHT were used, respectively. The upper, middle, and lower rows show cases when the RTVG values 5, 7, and 14 days after MHT were used, respectively. Note that the average MPI values in the control group were taken as zero. The red, blue, green, and black circles represent data in Groups A, B, and C and the control group, respectively.

apoptosis [21]. As shown in Figure 2, the temperature in the rectum was almost constant at approximately $35^{\circ} \mathrm{C}$ $5 \mathrm{~min}$ or more after the start of MHT, whereas the temperature in the tumor rose to approximately 41 to $44^{\circ} \mathrm{C}$ during MHT depending on the iron concentration of Resovist ${ }^{\circledR}$. These results suggest that the tumor was selectively heated by AMF. As shown in Figure 3, significant differences were observed in the RTVG value between 


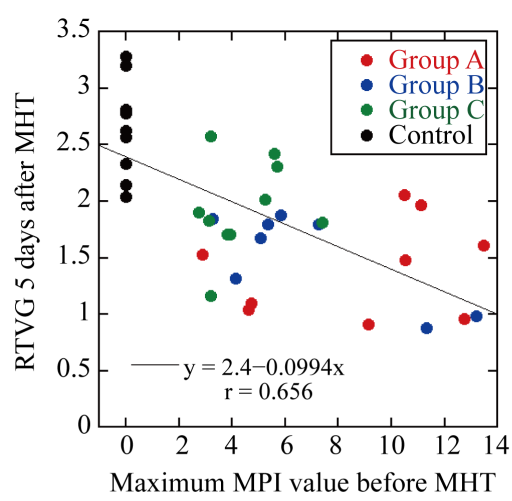

(a)

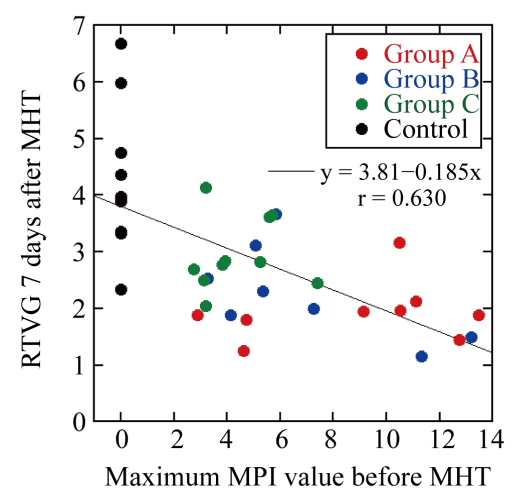

(c)

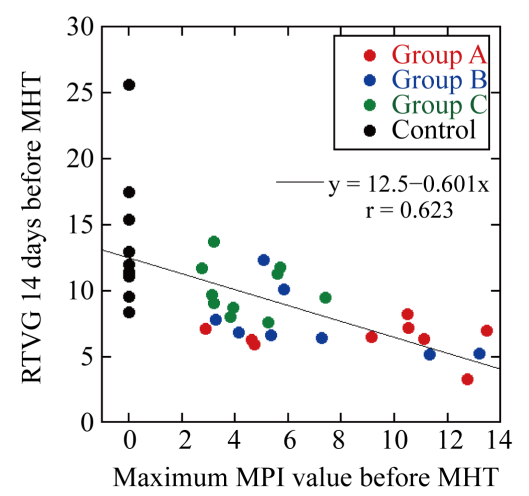

(e)

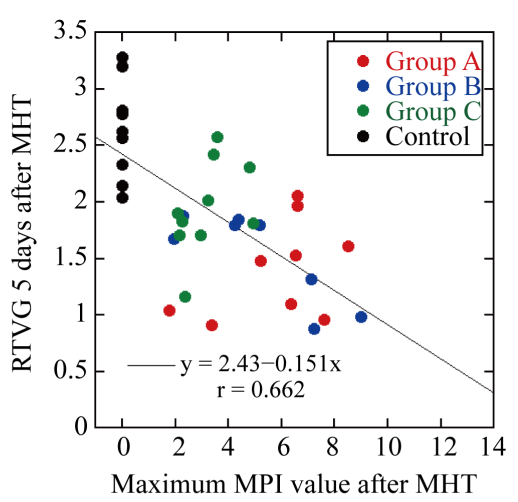

(b)

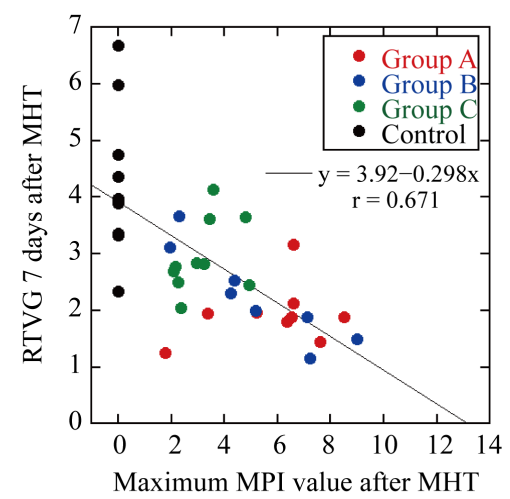

(d)

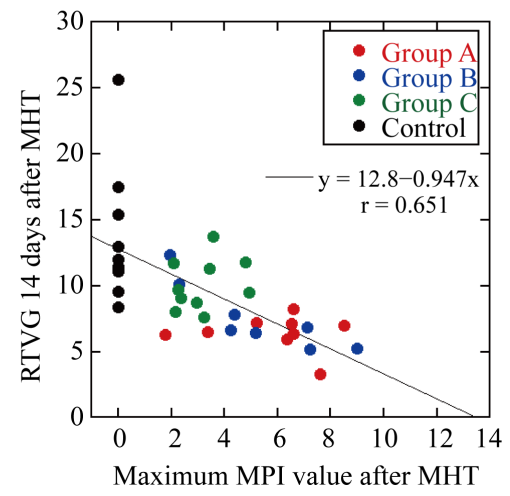

(f)

Figure 7. Correlation between the maximum MPI and RTVG values. The left and right columns show cases when the maximum MPI values immediately before and immediately after MHT were used, respectively. The upper, middle, and lower rows show cases when the RTVG values 5, 7, and 14 days after MHT were used, respectively. Note that the maximum MPI values in the control group were taken as zero. The red, blue, green, and black circles represent data in Groups A, B, and C and the control group, respectively.

Group A and the control group and between Group B and the control group. Although there was a tendency for the RTVG value in Group C to be lower than that in the control group, it did not reach statistical significance except at 5 days after MHT (Figure 3). Thus, it appears that the temperature of the tumor in Group C did not exceed $42^{\circ} \mathrm{C}$ to $43^{\circ} \mathrm{C}$, i.e., the threshold temperature for inducing cell death (Figure 2). These results suggest 


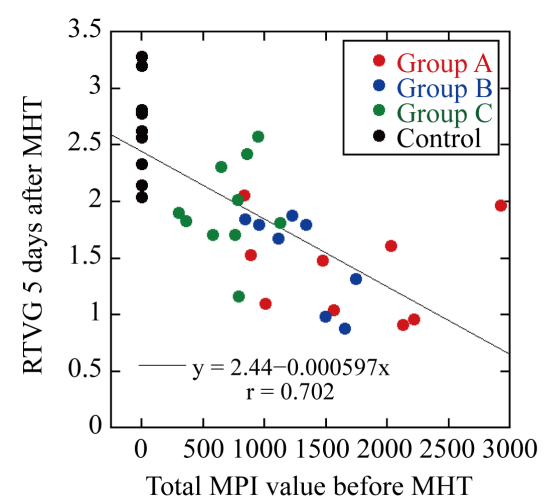

(a)

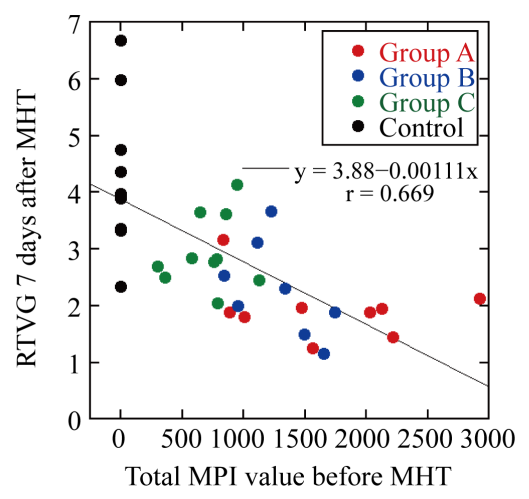

(c)

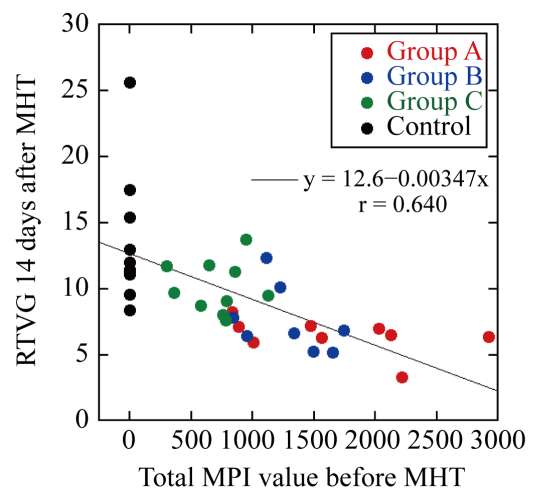

(e)

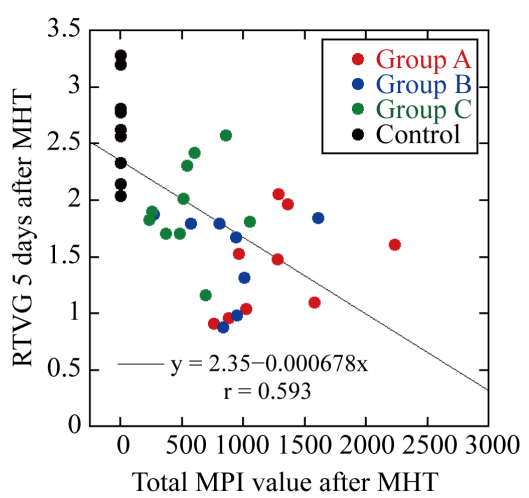

(b)

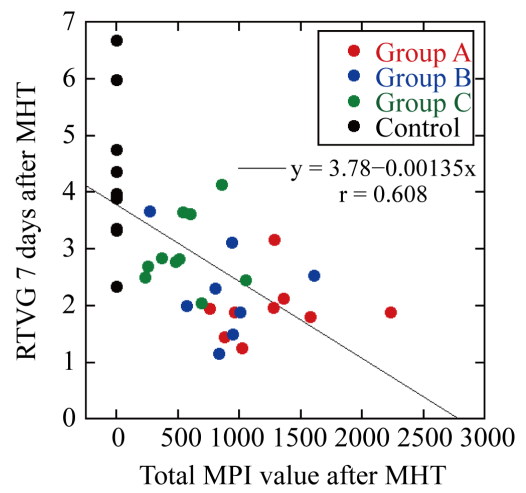

(d)

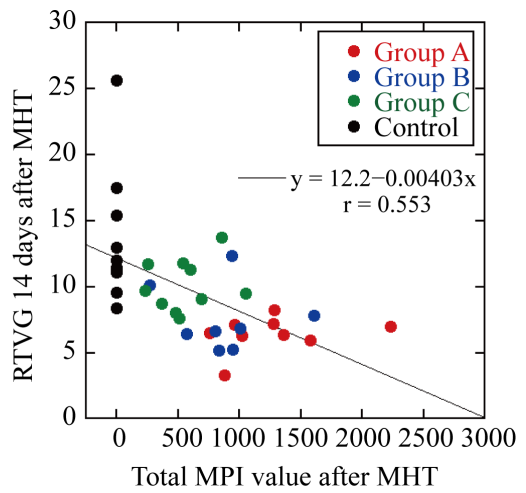

(f)

Figure 8. Correlation between the total MPI and RTVG values. The left and right columns show cases when the total MPI values immediately before and immediately after MHT were used, respectively. The upper, middle, and lower rows show cases when the RTVG values 5, 7, and 14 days after MHT were used, respectively. Note that the total MPI values in the control group were taken as zero. The red, blue, green, and black circles represent data in Groups A, B, and C and the control group, respectively.

that the therapeutic effect of MHT depends on the temperature of the tumor and therefore on the iron concentration of MNPs accumulated in the tumor. To enhance the therapeutic effect of MHT, it will be necessary to deliver and accumulate sufficient MNPs to tumors and/or to optimize the parameters of AMF such that the tumors are heated above $42^{\circ} \mathrm{C}$ to $43^{\circ} \mathrm{C}$. As previously described, the frequency and peak amplitude of AMF and the 


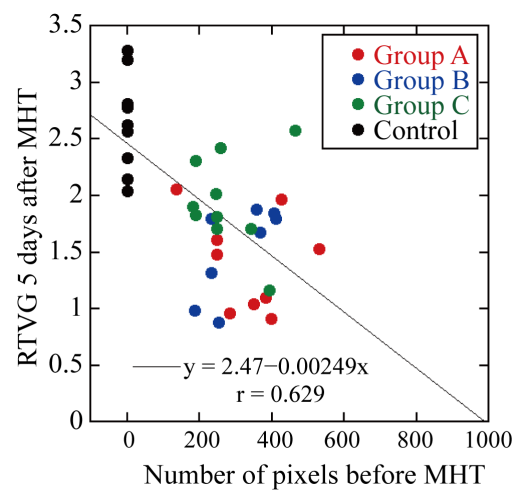

(a)

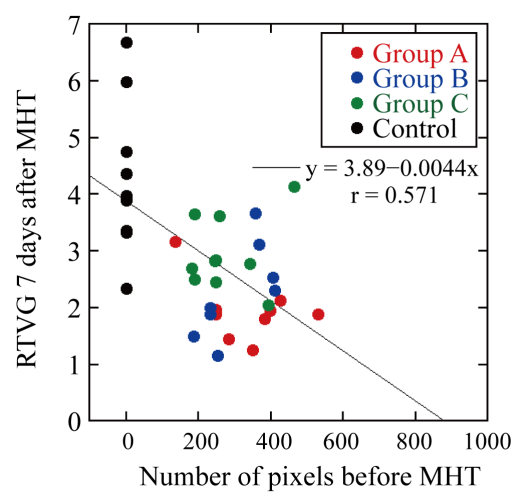

(c)

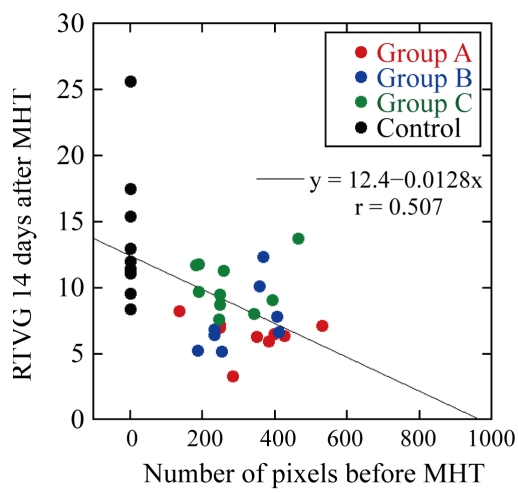

(e)

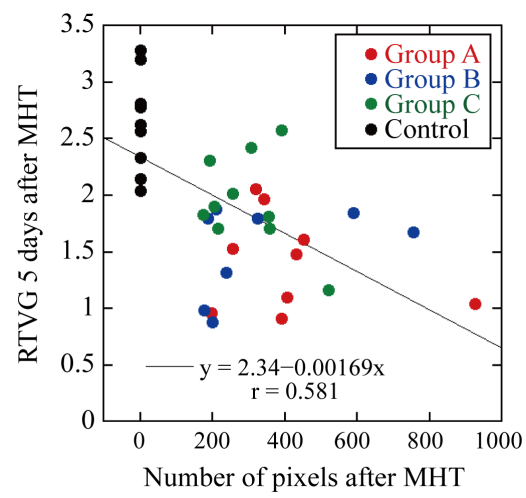

(b)

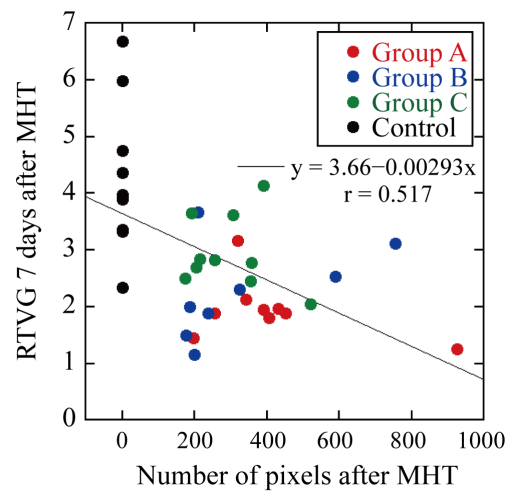

(d)

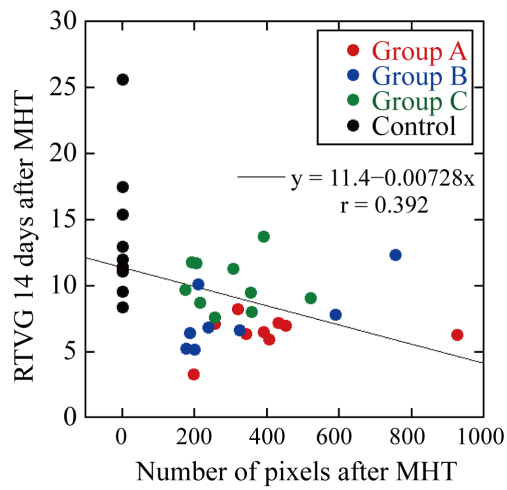

(f)

Figure 9. Correlation between the number of pixels within the ROI drawn on the tumor and the RTVG value. The left and right columns show cases when the numbers of pixels immediately before and immediately after MHT were used, respectively. The upper, middle, and lower rows show cases when the RTVG values 5, 7, and 14 days after MHT were used, respectively. Note that the number of pixels in the control group was taken as zero. The red, blue, green, and black circles represent data in Groups A, B, and C and the control group, respectively.

duration of MHT were set at $600 \mathrm{kHz}, 3.5 \mathrm{kA} / \mathrm{m}$, and $20 \mathrm{~min}$, respectively, in this study. It might be possible to improve the therapeutic effect in Group $\mathrm{C}$ by changing these parameters [22]. The frequency $(f)$ and peak amplitude $(H)$ of AMF, however, should be chosen under a criterion of $f \cdot H<5 \times 10^{9} \mathrm{Am}^{-1} \cdot \mathrm{s}^{-1}$ to prevent unwanted damage to the surrounding healthy tissue via eddy currents [23]. 
Since there is a significant correlation between the average MPI value and the temperature rise generated by MNPs, as we previously reported [15], the temperature in regions with a higher MPI value is considered to become higher than that in regions with a lower MPI value during MHT. If we could determine the MPI value at which the temperature of the tumor rises to $42^{\circ} \mathrm{C}$ to $43^{\circ} \mathrm{C}$, it would be possible to use this MPI value as an indicator for estimating the effectiveness of MHT. In this study, we directly injected MNPs into the tumor as previously described. It is known that the spatial distribution and amount of the MNPs accumulated in the tumor largely depend on the injection speed and/or actual injected dose of MNPs [24]. Thus, it would be necessary to quantify the amount of MNPs in the tumor accurately after the injection of MNPs in order to estimate the temperature rise in the tumor and thus to predict the therapeutic effect of MHT. Since there is an excellent linear correlation between the average MPI value and the iron concentration of Resovist ${ }^{\circledR}$ as we previously demonstrated by phantom studies [15], MPI will be useful for accurately quantifying the amount of MNPs in the tumor after MNP injection.

In this study, we investigated the temporal change of MNPs accumulated in the tumor by calculating the average, maximum, and total MPI values and the number of pixels within the ROI drawn on the tumor in the MPI image immediately before, immediately after, 7 days, and 14 days after MHT (Figure 5). It appears that the change in the average MPI value corresponds to that in the average amount of MNPs per voxel, i.e., the average concentration of MNPs, and the change in the total MPI value corresponds to that in the total amount of MNPs in the selected slice of the tumor, whereas the change in the number of pixels corresponds to that in the distributed area of MNPs. As shown in Figure 5, the average, maximum, and total MPI values decreased greatly between immediately before and immediately after MHT and between immediately after and 7 days after MHT, and did not largely change thereafter. In contrast, the number of pixels tended to increase with time. These findings suggest that the intratumorally injected MNPs were distributed locally near the injected site immediately after the injection of MNPs and dispersed within the tumor during MHT, and that the MNPs remained in the tumor thereafter. Once MNPs are injected, MPI can be performed repeatedly until the MNPs disappear. This knowledge of the temporal change of the concentration and spatial distribution of MNPs in the tumor obtained by the repeated MPI studies will be useful for the treatment planning of MHT. Especially, the fact that MNPs remain in the tumor even 7 and 14 days after MHT will be useful when considering the repeated application of MHT to enhance its therapeutic efficacy [25].

Kettering et al. [25] investigated the bio-distribution of intratumorally injected MNPs in mice focused on MNP long term monitoring of pre and post therapy over 7 days using multi-channel magnetorelaxometry (MRX). Furthermore, they investigated the bio-distribution of MNPs in internal organs and tumors of sacrificed animals using single-channel MRX, and reported that there was no distinct change of total MNP amounts during long term monitoring and most of the MNPs remained in the tumors; only a few MNPs were detected in the liver and spleen and less than $1 \%$ of the injected MNPs were excreted. Their findings, however, are limited to the wholebody or whole-organ amount of MNPs, because the local concentration and spatial distribution of MNPs in the tumor cannot be measured in vivo using multi-channel or single-channel MRX [25]. The temperature rise generated by MNPs in steady state is proportional to the concentration of MNPs [23]. When we design an optimal MHT treatment plan to prevent insufficient heating of the targeted region and overheating of the healthy tissue, accurate knowledge of the local concentration of MNPs accumulated in the targeted region appears to be more important than knowledge of the total amount of MNPs when the spatial distribution of MNPs is inhomogeneous.

As previously described, we started MHT 20 min after the injection of MNPs, whereas Kettering et al. [25] did so 24 hours after the injection. Giustini et al. [26] reported that the intracellular uptake and aggregation of MNPs varied depending on the duration after the injection of MNPs, which may exert an influence on the heat deposition in the tumor and tumor cytotoxicity when exposed to AMF. Thus, the start time of MHT might affect the spatial distribution and temporal change of MNPs in the tumor and the resulting therapeutic effect of MHT. Further studies on these aspects are currently in progress.

As shown in Figures 6-9, the average, maximum, and total MPI values and the number of pixels immediately before and immediately after MHT were significantly inversely correlated with the RTVG values 5, 7, and 14 days after MHT, suggesting that these parameters are useful for predicting the RTVG 5 to 14 days after MHT. However, there was a tendency for the correlation coefficients between the parameters immediately before MHT and the RTVG values to be higher than those when using the parameters immediately after MHT, except for 
cases of the maximum MPI value, suggesting that the parameters immediately before MHT may be more effective for predicting the therapeutic effect of MHT than those immediately after MHT. Furthermore, the correlation coefficients between the number of pixels immediately before or after MHT and the RTVG value tended to be lower than those when using the average, maximum, and total MPI values, suggesting that the number of pixels immediately before or after MHT is less effective for predicting the therapeutic effect of MHT than other parameters.

When we analyzed the statistical difference in the number of pixels among groups (Figure 5(d)), the number of combinations that reached statistical significance was lower than those in other parameters such as the average and maximum MPI values (Figure 5(a) and Figure 5(b)). This was also the case when we analyzed the statistical significance in the correlation between the number of pixels and the RTVG value (Figure 9) as described above. These results appear to be mainly due to the fact that the scatter of the data of this parameter is larger than those of other parameters. As previously described, when we drew an ROI on the tumor in the MPI image, we took the threshold value for extracting the contour of the tumor as $40 \%$ of the maximum MPI value within the ROI. The threshold value adopted in this study was determined visually from inspection of the MPI images with various threshold values, which were superimposed on the X-ray CT images. Although this threshold value appears to be appropriate in our experience, the number of pixels might be more susceptible to the selection of the threshold value or the method for determining an ROI than other parameters. Thus, further studies on the optimization of the threshold value or the method for determining an ROI may be necessary.

A limitation of this study is that the MPI value was obtained from a single slice of the MPI image with the maximum signal intensity. Analysis with use of a single slice of the MPI image limits the accurate evaluation of the spatial distribution of MNPs in the whole tumor. For more detailed analysis, it will be necessary to acquire three-dimensional (multi-slice) data and to evaluate the three-dimensional distribution and accumulation of MNPs from these data. If this can be realized in the future, we expect that our MPI system can be used for more precise diagnosis and prediction of the therapeutic effect of MHT and can be applied to theranostics, in which diagnosis and therapy are integrated in a single platform. These studies are also currently in progress.

Other methods for imaging MNPs are magnetic resonance imaging (MRI) and micro-CT imaging. When we attempted to image MNPs using MRI with a conventional transverse relaxation time $\left(T_{2}{ }^{*}\right)$-weighted imaging sequence, it was almost impossible due to large susceptibility-induced MR signal loss and image distortions in the regions near the MNPs especially for the higher concentrations of MNPs as is the case in MHT. Recently, Dähring et al. [27] proposed the use of micro-CT for determining the MNP distributions within tumors and reported that the information about the MNP distribution obtained by micro-CT permitted individualized MHT and improved the overall therapeutic efficacy. Although the use of micro-CT also appears to be promising and useful for establishing effective MHT, further studies especially on the accuracy and reproducibility in quantifying the amount of MNPs might be necessary for establishing the usefulness of the method.

\section{Conclusion}

We could visualize intratumorally injected MNPs in mice using our MPI system and could quantitatively evaluate the temporal change of the spatial distribution of MNPs in tumors. We found that the intratumorally injected MNPs dispersed within the tumor during MHT and then remained in the tumor even 7 and 14 days after MHT. In addition, we found that the MPI values immediately before or immediately after MHT were significantly inversely correlated with the RTVG values. These results suggest that MPI allows us to quantitatively evaluate the temporal change of the spatial distribution of MNPs in vivo in a visible manner and will be useful for predicting the therapeutic effect of MHT and for MHT treatment planning.

\section{Acknowledgements}

This work was supported by a Grant-in-Aid for Scientific Research (B) (Grant Number: 25282131) from the Japan Society for the Promotion of Science (JSPS).

\section{Declaration of Interest}

The authors report no conflicts of interest. The authors alone are responsible for the content and writing of the paper. 


\section{References}

[1] Abe, M., Hiraoka, M., Takahashi, M., Egawa, S., Matsuda, C., Onoyama Y, Morita, K., Kakehi, M. and Sugahara, T. (1986) Multi-Institutional Studies on Hyperthermia Using an 8-MHz Radiofrequency Capacitive Heating Device (Thermotron RF-8) in Combination with Radiation for Cancer Therapy. Cancer, 58, 1589-1595. http://dx.doi.org/10.1002/1097-0142(19861015)58:8<1589::AID-CNCR2820580802>3.0.CO;2-B

[2] Overgaard, J. (1980) Simultaneous and Sequential Hyperthermia and Radiation Treatment of an Experimental Tumor and Its Surrounding Normal Tissue in Vivo. International Journal of Radiation Oncology, Biology, Physics, 6, 15071517. http://dx.doi.org/10.1016/0360-3016(80)90008-5

[3] Ito, A., Honda, H. and Kobayashi, T. (2006) Cancer Immunotherapy Based on Intracellular Hyperthermia Using Magnetite Nanoparticles: A Novel Concept of "Heat-Controlled Necrosis" with Heat Shock Protein Expression. Cancer Immunology, Immunotherapy, 55, 320-328. http://dx.doi.org/10.1007/s00262-005-0049-y

[4] Haas, G.P., Klugo, R.C., Hetzel, F.W., Barton, E.E. and Cerny, J.C. (1984) The Synergistic Effect of Hyperthermia and Chemotherapy on Murine Transitional Cell Carcinoma. The Journal of Urology, 132, 828-833.

[5] Matsumoto, K., Yamamoto, N., Hagiwara, S., Saito, M., Furue, H., Shigetomi, T., Narita, Y., Mitsudo, K., Tohnai, I., Kobayashi, T. and Ueda, M. (2011) Optimization of Hyperthermia and Dendritic Cell Immunotherapy for Squamous Cell Carcinoma. Oncology Reports, 25, 1525-1532.

[6] Van der Zee, J. (2002) Heating the Patient: A Promising Approach? Annals of Oncology, 13, 1173-1184. http://dx.doi.org/10.1093/annonc/mdf280

[7] Murase, K., Oonoki, J., Takata, H., Song, R., Angraini, A., Ausanai, P. and Matsushita, T. (2011) Simulation and Experimental Studies on Magnetic Hyperthermia with Use of Superparamagnetic Iron Oxide Nanoparticles. Radiological Physics and Technology, 4, 194-202. http://dx.doi.org/10.1007/s12194-011-0123-4

[8] Petryk, A.A., Giustini, A.J., Gottesman, R.E., Trembly, B.S. and Hoopes, P.J. (2013) Comparison of Magnetic Nanoparticle and Microwave Hyperthermia Cancer Treatment Methodology and Treatment Effect in a Rodent Breast Cancer. International Journal of Hyperthermia, 29, 819-827. http://dx.doi.org/10.3109/02656736.2013.845801

[9] Kumar, C.S. and Mohammad, F. (2011) Magnetic Nanomaterials for Hyperthermia-Based Therapy and Controlled Drug Delivery. Advanced Drug Delivery Reviews, 63, 789-808. http://dx.doi.org/10.1016/j.addr.2011.03.008

[10] Hardiansyah, A., Huang, L.Y., Yang, M.C., Liu, T.Y., Tsai, S.C., Yang, C.Y., Kuo, C.Y., Chan, T.Y., Lian, W.N. and Lin, C.H. (2014) Magnetic Liposomes for Colorectal Cancer Cells Therapy by High-Frequency Magnetic Field Treatment. Nanoscale Research Letters, 9, 497. http://dx.doi.org/10.1186/1556-276X-9-497

[11] Kawai, N., Ito, A., Nakahara, Y., Honda, H., Kobayashi, T., Futakuchi, M., Shirai, T., Tozawa, K. and Kohri K. (2006) Complete Regression of Experimental Prostate Cancer in Nude Mice by Repeated Hyperthermia Using Magnetite Cationic Liposomes and a Newly Developed Solenoid Containing a Ferrite Core. Prostate, 66, 718-727. http://dx.doi.org/10.1002/pros.20394

[12] Shinkai, M., Suzuki, M., Iijima, S. and Kobayashi, T. (1995) Antibody-Conjugated Magnetoliposomes for Targeting Cancer Cells and Their Application in Hyperthermia. Biotechnology and Applied Biochemistry, 21, 125-137. http://dx.doi.org/10.1111/j.1470-8744.1995.tb00329.x

[13] Gleich, B. and Weizenecker, J. (2005) Tomographic Imaging Using the Nonlinear Response of Magnetic Particles. Nature, 435, 1214-1217. http://dx.doi.org/10.1038/nature03808

[14] Murase, K., Hiratsuka, S., Song, R. and Takeuchi, Y. (2014) Development of a System for Magnetic Particle Imaging Using Neodymium Magnets and Gradiometer. Japanese Journal of Applied Physics, 53, Article ID: 067001. http://dx.doi.org/10.7567/jjap.53.067001

[15] Murase, K., Aoki, M., Banura, N., Nishimoto, K., Mimura, A., Kuboyabu, T. and Yabata, I. (2015) Usefulness of Magnetic Particle Imaging for Predicting the Therapeutic Effect of Magnetic Hyperthermia. Open Journal of Medical Imaging, 5, 85-99. http://dx.doi.org/10.4236/ojmi.2015.52013

[16] Murase, K., Banura, N., Mimura, A. and Nishimoto, K. (2015) Simple and Practical Method for Correcting the Inhomogeneous Sensitivity of a Receiving Coil in Magnetic Particle Imaging. Japanese Journal of Applied Physics, 54, Article ID: 038001.

[17] Murase, K., Song, R. and Hiratsuka, S. (2014) Magnetic Particle Imaging of Blood Coagulation. Applied Physics Letters, 104, Article ID: 252409. http://dx.doi.org/10.1063/1.4885146

[18] Nishimoto, K., Mimura, A., Aoki, M., Banura, N. and Murase, K. (2015) Application of Magnetic Particle Imaging to Pulmonary Imaging Using Nebulized Magnetic Nanoparticles. Open Journal of Medical Imaging, 5, 49-55. http://dx.doi.org/10.4236/ojmi.2015.52008

[19] Murase, K., Mimura, A., Banura, N., Nishimoto, K. and Takata, H. (2015) Visualization of Magnetic Nanofibers Using Magnetic Particle Imaging. Open Journal of Medical Imaging, 5, 56-65. http://dx.doi.org/10.4236/ojmi.2015.52009 
[20] Yonezawa, M., Otsuka, T., Matsui, N., Tsuji, H., Kato, K.H., Moriyama, A. and Kato, T. (1996) Hyperthermia Induces Apoptosis in Malignant Fibrous Histiocytoma Cells in Vitro. International Journal of Cancer, 66, 347-351. http://dx.doi.org/10.1002/(SICI)1097-0215(19960503)66:3<347::AID-IJC14>3.0.CO;2-8

[21] Bettaieb, A. and Averill-Bates, D.A. (2015) Thermotolerance Induced at a Mild Temperature of $40^{\circ} \mathrm{C}$ Alleviates Heat Shock-Induced ER Stress and Apoptosis in Hela Cells. Biochimica et Biophysica Acta, 1853, 52-62. http://dx.doi.org/10.1016/j.bbamcr.2014.09.016

[22] Kang, M.S., Song, C.W. and Levitt, S.H. (1980) Role of Vascular Function in Response of Tumors in Vivo to Hyperthermia. Cancer Research, 40, 1130-1135. http://dx.doi.org/10.1016/j.jmmm.2006.10.1156

[23] Hergt, R. and Dutz, S. (2007) Magnetic Particle Hyperthermia-Biophysical Limitations of a Visionary Tumour Therapy. Journal of Magnetism and Magnetic Materials, 311, 187-192.

[24] Javidi, M., Heydrari, M., Karimi, A., Haghpanahi, M., Navidbakhsh, M. and Razmkon, A. (2014) Evaluation of the Effects of Injection Velocity and Different Gel Concentrations on Nanoparticles in Hyperthermia Therapy. Journal of Biomedical Physics and Engineering, 4, 151-162.

[25] Kettering, M., Richter, H., Wiekhorst, F., Bremer-Streck, S., Trahms, L., Kaiser, W.A. and Hilger, I. (2011) MinimalInvasive Magnetic Heating of Tumors Does Not Alter Intra-Tumoral Nanoparticle Accumulation, Allowing for Repeated Therapy Sessions: An in Vivo Study in Mice. Nanotechonogy, 22, Article ID: 505102. http://dx.doi.org/10.1088/0957-4484/22/50/505102

[26] Giustini, A.J., Ivkov, R. and Hoopes, P.J. (2011) Magnetic Nanoparticle Biodistribution Following Intratumoral Administration. Nanotechnology, 22, Article ID: 345101. http://dx.doi.org/10.1088/0957-4484/22/34/345101

[27] Dähring, H., Grandke, J., Teichgräber, U. and Hilger, I. (2015) Improved Hyperthermia Treatment of Tumors under Consideration of Magnetic Nanoparticle Distribution Using Micro-CT Imaging. Molecular Imaging and Biology, 17, 763-769. http://dx.doi.org/10.1007/s11307-015-0848-2 